\title{
Nanopartículas de plata funcionalizadas in situ con D-limoneno: efecto en la actividad antibacteriana
}

\author{
Julián Echeverry-Chica', 2; Andrea Naranjo-Díaz; Pedronel Araque-Marín ${ }^{1 *}$ \\ ${ }^{1}$ Grupo de Investigación e Innovación en Formulaciones Químicas, Escuela de Ciencias de la Vida, Universidad \\ EIA, km 2+200 vía al aeropuerto José María Córdova, Envigado, Colombia. \\ 2Laboratorio Clínico Hematológico. Carrera 43c No 5 - 33, Medellín, Colombia. \\ *pedronel.araque@eia.edu.co
}

Fecha recepción: octubre 15 de 2019 Fecha aceptación: enero 17 de 2020

\begin{abstract}
Resumen
Este estudio se centró en la formulación y caracterización de nanopartículas de plata (AgNP) funcionalizadas con d-limoneno. Las nanopartículas se funcionalizaron por inversión de fase y la síntesis de las nanopartículas se realizó in situ; se determinó el tamaño de partícula por difracción laser, potencial zeta y estabilidad coloidal óptica utilizando Multiscan 20 por un periodo de 24 horas a $37^{\circ} \mathrm{C}$; se determinó la concentración mínima inhibitorio (CMI) y la concentración mínima bactericida (CMB) del material formulado sobre las bacterias Escherichia coli ATCC 25922, Staphylococcus aureus ATCC 29213, Klebsiella oxytoca ATCC 700324, Enterococcus casseliflavus ATCC 700327, Escherichia coli BLEE+, Pseudomona aeruginosa resistente a carbapenémicos. Las nanopartículas presentaron estabilidad coloidal a una concentración de d-limoneno del 3,93 \%, iones plata al 1,61 $\mathrm{x}^{-3} \%$, coadyuvante no iónico al $24 \%$ y ácido ascórbico al 5,88 \%; cómo sistema regulador se utilizó ácido cítrico/citrato (1:1) 0,48M para un $\mathrm{pH}$ de 4,5. La formulación se clasificó como un sistema polidisperso ( $P D=0,0851$ ), con una potencial zeta de $-11,6 \mathrm{mV}$ y tamaño promedio de partícula de $81,5 \pm 0,9 \mathrm{~nm}$. Se evidenció una velocidad de migración de partícula de $-0,199 \pm 0,006 \mathrm{~mm} \mathrm{~h}^{-1}$, un perfil de transmisión constante y perfil de retrodispersión con variaciones del $10 \%$, lo que representa una formulación estable. Las nanopartículas presentaron una CMl y una CMB de $28 \mathrm{\mu g} \mathrm{mL}^{-1}\left(5,6 \times 10^{-2} \%\right.$ d-limoneno y $\left.4,7 \times 10^{-5} \% \mathrm{AgNP}\right)$ contra todas las bacterias probadas.
\end{abstract}

Palabras clave: Nanopartículas de Plata; Inversión de Fases; Resistencia Bacteriana; Concentración Mínima Inhibitoria. 


\title{
Silver nanoparticles functionalized in situ with D-Limonene: effect on antibacterial activity
}

\begin{abstract}
This study focused on the formulation and characterization of silver nanoparticles (AgNP) functionalized with $\mathrm{d}$-limonene. The nanoparticles were functionalized by phase inversion and the synthesis of the nanoparticles was performed in situ; the particle size was determined by laser diffraction, zeta potential and colloidal optical stability using Multiscan 20 for a period of 24 hours at $37^{\circ} \mathrm{C}$; the minimum inhibitory concentration (MIC) and the minimum bactericidal concentration (CMB) of the formulated material on the bacterias Escherichia coli ATCC 25922, Staphylococcus aureus ATCC 29213, Klebsiella oxytoca ATCC 700324, Enterococcus casseliflavus ATCC 700327, Escherichia coli BLEE, Pseudomona aeruginosa resistant to carbapenems. The nanoparticles showed colloidal stability at a concentration of $3.93 \%$ d-limonene, $1.6 \times 10^{-3} \%$ silver ions, $24 \%$ non-ionic adjuvant and $5.88 \%$ ascorbic acid, citric acid/citrate was used as a regulatory system (1:1) $0.48 \mathrm{M}$ for a pH of 4.5. The formulation was classified as a polydispersed system $(P D=0.0851)$, with a zeta potential of $-11.6 \mathrm{mV}$ and average particle size of $81.5 \pm 0.9 \mathrm{~nm}$. A particle migration rate of $-0.199 \pm 0.006 \mathrm{~mm} \mathrm{~h}^{-1}$ was evidenced, a constant transmission profile, a backscatter profile with variations of $10 \%$, which represents a stable formulation. The nanoparticles presented an MIC and an MIB of $28 \mu \mathrm{g} \mathrm{mL}^{-1}\left(5.6 \times 10^{-2} \%\right.$ limonene, $\left.4.7 \times 10^{-5} \% \mathrm{AgNP}\right)$ against all the bacteria tested.
\end{abstract}

Keywords: Silver Nanoparticles; Phase Inversion; Bacterial Resistance; Minimal Inhibitory Concentration.

\section{Nanopartículas de prata funcionalizadas in situ com D-Limoneno: efeito na atividade antibacteriana}

\begin{abstract}
Resumo
Este estudo focou na formulação e caracterização de nanopartículas de prata AgNP funcionalizadas com d-limoneno. As nanopartículas foram funcionalizadas por inversão de fase e a síntese das nanopartículas foi realizada in situ; o tamanho das partículas foi determinado por difração a laser, potencial zeta e estabilidade óptica coloidal usando o Multiscan 20 por um período de 24 horas a $37^{\circ} \mathrm{C}$; a concentração inibitória mínima (CMI) e a concentração bactericida mínima (CMB) do material formulado na bactéria Escherichia coli ATCC 25922, Staphylococcus aureus ATCC 29213, Klebsiella oxytoca ATCC 700324, Enterococcus casseliflavus ATCC 700327, Escherichia coli BLEE, Pseudomona aeres. As nanopartículas

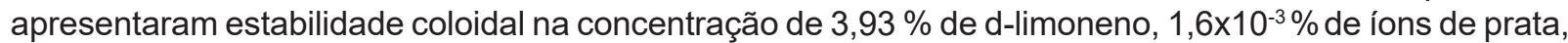
$24 \%$ de adjuvante não iônico e 5,88 \% de ácido ascórbico, ácido cítrico/ citrato (1:1) 0,48 M foi usado como sistema regulador para um $\mathrm{pH}$ de 4,5. A formulação foi classificada como um sistema polidisperso $(P D=0,0851)$, com um potencial zeta de $-11,6 \mathrm{mV}$ e tamanho médio de partícula de 81,5 $\pm 0,9 \mathrm{~nm}$. Era evidente uma taxa de migração de partículas de $-0,199 \pm 0,006 \mathrm{~mm} \mathrm{~h}^{-1}$, um perfil de transmissão constante, um perfil de retroespalhamento com variações de $10 \%$, o que representa uma formulação estável. A nanopartículas apresentou CIM e MIB de 28 mg mL-1 (d-limoneno 5,6x10-2 \%, AgNP 4,7×10-5\%) contra todas as bactérias testadas.
\end{abstract}

Palavras-chave: Nanopartículas de Prata; Inversão de Fase; Resistência Bacteriana; Concentração Inibitória Mínima. 


\section{Introducción}

Debido al uso indiscriminado de compuestos químicos artificiales como antibióticos y desinfectantes, para el tratamiento de enfermedades infecciosas en el ser humano y otras especies, para la producción pecuaria, para la limpieza y desinfección de ambientes, para la producción y conservación de alimentos, muchos de los microorganismos expuestos desarrollan rápidamente resistencia a estos compuestos, convirtiéndose en un problema de salud pública a nivel mundial al generar infecciones que no se pueden tratar; adicionalmente, los compuestos artificiales pueden causar directamente enfermedades al ser humano y otros seres vivos y contaminar el medio ambiente.

La Organización Mundial de la Salud OMS, en el año 2017 señaló que la batería de antimicrobianos disponibles para combatir bacterias multirresistentes como Pseudomonas aeruginosa, Acinetobacter baumannii, Mycobacterium tuberculosis y Staphylococcus aureus, es insuficiente para mitigar su proliferación y causar infección en seres humanos; se requiere de manera urgente el diseño de productos innovadores, que permitan controlar microorganismos causantes de enfermedad, que ponen en peligro la vida del ser humano y incrementan los costos de los tratamientos [1-6]. Existe una creciente tendencia a la investigación y utilización de extractos naturales $O$ aceites esenciales (AE) que han demostrado in vitro la capacidad de inhibir el crecimiento de bacterias de importancia clínica, como agentes antibióticos o desinfectantes [4, $5,44]$. Adicionalmente, ha cobrado importancia el desarrollo de nuevas formulaciones utilizando compuestos inorgánicos, siendo protagonistas los iones metálicos, conocidos por ser muy tóxicos para las células bacterianas $[3,7]$. nanopartículas metálicas presentan un mejor desempeño para eliminar bacterias al aumentar el área de superficie para reaccionar $[3,7,8]$. La comunidad científica debe aportar a la solución de la problemática mediante la investigación y la selección cuidadosa de agentes activos y formulaciones que se dirigen a múltiples sitios bacterianos y presenten modos de acción menos propensos a conferir resistencia cruzada.

La resistencia a los antimicrobianos se define como la adquisición de resistencia por parte de un microorganismo a un medicamento antimicrobiano al que anteriormente era sensible. La adquisición de resistencias por parte de las bacterias supone una amenaza para la salud pública; la mayor preocupación es la creciente propagación de bacterias patógenas multirresistentes a nivel mundial, debido al mal uso y abuso de los antibióticos $[9,10]$. Dentro de los mecanismos que expresan las bacterias para resistir a los varios grupos de antibióticos, está la producción de enzimas como las betalactamasas de espectro extendido (BLEE) que les confiere resistencia a oximino-cefalosporinas y a monobactámicos (aztreonam), antibióticos que actúan inhibiendo la síntesis de la pared celular bacteriana. Cuando una bacteria se identifica como productora de BLEE, como alternativa terapéutica se utilizan un grupo de antibióticos denominados carbapenems; estos actúan sobre la pared celular y poseen una gran resistencia a la hidrólisis frente a las BLEE [11]. Pero las bacterias también han creado múltiples mecanismos para evitar la acción de los carbapenems, convirtiéndose en una amenaza para la salud mundial, ya que por muchos años han sido los antibióticos más estables y activos contra bacterias con resistencia múltiple [12].

Las plantas del genero Citrus tienen altas concentraciones de AE en la cascara de sus frutos; su principal componente es el terpeno d-limoneno, molécula con reconocido poder inhibitorio del crecimiento de bacterias in vitro. En Colombia, los cítricos son considerados como la segundad especie frutícola que se produce después del banano, existiendo en Colombia suficiente materia prima para la obtención de AE de alto contenido en limoneno [4, 13-16]. Se ha reportado la actividad antimicrobiana de d-limoneno sobre el crecimiento in vitro de Streptococcus uberis, Sthapylococcus aureus, Staphylococcus epidermidis, Klebsiella pneumoniae, Pseudomonas fragi, Pseudomonas aeruginosa, Escherichia coli, Salmonella typhimurium, Salmonella enteritidis y Listeria monocytogenes [13, 15, 17-20]. Estos resultados demuestran las ventajas potenciales del uso de d-limoneno como antimicrobiano de origen natural. De los compuestos metálicos, la plata " $\mathrm{Ag}_{(\mathrm{s})}$ ", un elemento común en la naturaleza, ha sido utilizada por el ser humano como agente desinfectante [3, 21-23]. Las Las nanopartículas de plata (AgNP) tienen un fuerte potencial bactericida debido a su mayor relación superficie-volumen, presentando en promedio un tamaño de 10-100 nm, y al ser moléculas altamente reactivas se pueden incorporar como principio activo de medicamentos y desinfectantes, ofreciendo ventajas distintivas 
como la reducción de la toxicidad, la superación de la resistencia y la reducción del costo en comparación con los antibióticos convencionales $[2-4,7,24]$. Las AgNP pueden actuar como agentes antimicrobianos contra casi 650 especies, incluidas bacterias resistentes a antibióticos [5, 21], presentan un buen desempeño in vitro frente a bacterias Gram positivas como Staphylococcus aureus, Streptococcus pyogenes y Bacillus subtilis y Gram negativas como Pseudomonas aeruginosa, Escherichia coli y Salmonella typhi [2, 3, 7, 21 25]. Los mecanismos de acción de las AgNP sobre las bacterias inician con la unión a la membrana celular, proteínas de membrana y los ácidos nucleicos cargados negativamente, bloquean las cadenas respiratorias, generando especies reactivas de oxígeno, que conducen a cambios funcionales en la célula, hasta destruirla [4, 3, 21$23,25]$. Por otro lado, algunos autores reportan cepas bacterianas que presentan mecanismos de adaptación y/o resistencia a AgNP [21, 23].

En recientes estudios, se ha demostrado que las nanopartículas funcionalizadas con aceites esenciales aumentan su efecto antibacteriano y su biocompatibilidad $[4,5,7,41]$; se han evaluado los efectos antibacterianos de AgNP en combinación con el AE de la planta Zataria multiflora, observando que las AgNP con $A E$ presentan efecto sinérgico contra el crecimiento de Staphylococcus epidermidis y Staphylococcus aureus. Sin embargo, no se encontraron reportes que indiquen el potencial inhibitorio del crecimiento de bacterias, de formulados que combinen el d-limoneno y AgNP; en este estudio se propone producir una nanoemulsión que contenga AgNP funcionalizadas con d-limoneno y evaluar su efecto sobre actividad antibacteriana, esperando obtener un formulado que ofrezca una alternativa para el control y erradicación de agentes bacterianos de importancia clínica, especialmente microorganismos que manifiestan mecanismos para evitar la acción de los antibióticos y desinfectantes que se usan en la actualidad.

\section{Metodología}

A continuación se describeel proceso de formulación, caracterización y evaluación microbiológica de las AgNp funcionalizadas con d-linomeno.

\section{Formulación}

Nitrato de plata (>99 \%), ácido ascórbico (>99 \%), ácido cítrico (>99,5\%), citrato de sodio (>99 \%) y Tween $20 ®$ (para síntesis) fueron importados por Sigma-Aldrich Co (St. Louis, MO); la Glicerina al $87 \%$ por Merck y el alcohol etílico industrial al $96 \%$ por Químicos JM S.A. El d-limoneno fue donado por la Fundación de Apoyo a la Investigación en el Grupo Interdisciplinario de Estudios MolecularesFUNDAGIEM de la Universidad de Antioquia. Las formulaciones fueron preparadas en tubo Falcon de $50 \mathrm{~mL}$, adicionando coadyuvante no iónico (Tween 20®; Glicerina y alcohol etílico) y d-limoneno con agitación continua; luego, se adicionó lentamente solución de nitrato de plata y la mezcla sólida compuesta por ácido cítrico/ citrato de sodio. Posteriormente se adicionó lentamente ácido ascórbico con agitación continua en Vortex (Thermo Scientific) para la reducción del ion plata; se completó el volumen a $50 \mathrm{~mL}$ con agua desionizada.

La formulación F1 representa las AgNP funcionalizadas con d-limoneno, la formulación F2 representa AgNP sin adición de limoneno y la formulación F3 representa las nanoemulsión con d-limoneno y ausencia de AgNP.

\section{Análisis de tamaño de partícula y potencial z}

Las formulaciones fueron diluidas 1:10 con agua estéril inyectable. Posteriormente se analizaron en el NanoSight 300 de Malvern, el cual usa una técnica ideal para sistemas polidispersos y arroja un análisis por rastreo de partículas, permitiendo caracterizar nanopartículas de $10 \mathrm{~nm}$ a $2000 \mathrm{~nm}$.

\section{Análisis de estabilidad coloidal}

La estabilidad coloidal de las nanosuspensiones fue evaluada por el Multiscan 20 de DataPhysics. Se almacenó cada formulación en botellas cerradas de vidrio transparentes de $40 \mathrm{~mm}$, donde los productos se sometieron a análisis periódicos a $37^{\circ} \mathrm{C}$ durante 24 horas. El objetivo fue de acelerar los procesos de desestabilización y para detectar productos potencialmente inestables en la etapa más temprana posible para, en consecuencia, reducir el tiempo para el desarrollo de nuevos productos.

\section{Análisis microbiológico}

Para las pruebas de la actividad antibacteriana se utilizaron las bacterias Escherichia coli ATCC 25922, Staphylococcus aureus ATCC 29213, Klebsiella oxytoca ATCC 700324, Enterococcus casseliflavus ATCC 700327, Escherichia coli productora de BLEE, Pseudomona aeruginosa que 
presentó impermeabilidad a los carbapenémicos, estas dos últimas aisladas a partir de muestras de orina. Todas cepas fueron proporcionadas por el Laboratorio Clínico Hematológico. Las bacterias crioconservadas en agar Infusión cerebro corazón $\mathrm{BH}$, glicerol y suero bovino fetal, se descongelan y los bacilos Gram negativos se siembran por agotamiento en agar MacConkey (Biomériux) y los cocos Gram positivos en agar Columbia CNA Biomériux. Se incubaron durante 24 horas a $37^{\circ} \mathrm{C}$; se identificó el género y la especie de cada crecimiento por medio del equipo Vitek 2 Compac Biomériux. Una vez confirmado el género y la especie, se procedió a la determinación de la CMI.

\section{Método de dilución en agar}

Este método permitió cuantificar la actividad in vitro de un antimicrobiano por medio de la determinación del crecimiento de un microorganismo en una serie de diluciones del antibiótico en mezcla con medio de cultivo. El método de dilución en agar permitió determinar la concentración mínima bactericida $\mathrm{CMB}$, definida como la concentración más baja del agente antimicrobiano necesaria para eliminar el 99 \% del inoculo inicial y la concentración mínima inhibitoria $\mathrm{CMI}$, definida como la concentración más baja de sustancia que puede inhibir el crecimiento visible de un microorganismo. Para realizar las diluciones del producto antibacteriano en estudio se dispersaron en un Erlenmeyer la cantidad del agente antibacteriano a analizar, posteriormente es agregada una cantidad conocida de agar estéril aún fundido $\left(50^{\circ} \mathrm{C}\right)$; se suele utilizar el agar Müeller-Hinton que permite el desarrollo de bacilos Gram negativos y de cocos Gram positivos. Se homogeniza la mezcla y se vierte en una placa de Petri estéril vacía, obteniendo de esta manera una placa de agar Müeller-Hinton con el antibiótico diluido a una determinada concentración lista para ser inoculada [11, 26-29]. La CMI fue aquella dilución en la cual no se observa crecimiento de la bacteria probada. La CMB se establece tomando una muestra con un hisopo estéril de la superficie del agar sólido que contiene la dilución que permitió establecer la $\mathrm{CMI}$ y se procede a cultivar la muestra en un agar Müeller-Hinton. Se incuba durante 48 horas a $37^{\circ} \mathrm{C}$, esperando que no se observe crecimiento bacteriano [26]. El Agar Müeller-Hinton es un medio sólido, no selectivo, que permite la realización de las pruebas de susceptibilidad antimicrobiana en bacterias aerobia, anaerobias y microaerofilicas [28]. Tiene un poder inhibitorio bajo y alta reproducibilidad; debe estar preparado a un $\mathrm{pH}$ entre 7,2 y 7,4 que puede ser ajustado con $\mathrm{Ca}^{2+}\left(20-25 \mathrm{mg} \mathrm{L}^{-1}\right)$ y $\mathrm{Mg}^{2+}$ (10-12,5 $\left.\mathrm{mg} \mathrm{L}^{-1}\right)$ [29].

\section{Determinación de la Concentración Mínima Inhibitoria (CMI)}

La CMI de los formulados F1, F2, F3 se determinó por el método de dilución en agar descrito en la guía CLSI M-7 2018 [30], con algunas modificaciones; después de 24 horas de incubación a $37^{\circ} \mathrm{C}$, se toman tres a cuatro colonias de cada bacteria con un palillo de madera estéril y se suspenden en solución salina al $0,85 \%$, hasta alcanzar un patrón de turbidez de $0,5 \mathrm{McF}$ arland $\left(1,5 \times 10^{8} \mathrm{UFC} \mathrm{mL}^{-1}\right)$. Se comprueba cada suspensión con el equipo Densichek (Biomériux). Se utilizó agar MuellerHinton (Becton Dickinson, USA) para realizar las diluciones, incorporando la cantidad determinada de la nanoemulsión en el agar cuando aún se encuentra en fase líquida $\left(50{ }^{\circ} \mathrm{C}\right)$, conservando siempre un volumen final de $10 \mathrm{~mL}$; la primera dilución contiene $1 \mathrm{~mL}$ de formulado $+9 \mathrm{~mL}$ de agar, la dilución final contiene $100 \mu \mathrm{l}$ de formulado + $900 \mu \mathrm{l}$ de agua destilada estéril $+9 \mathrm{~mL}$ de agar. Se deposita la mezcla en placas Petris plásticas estériles y se deja enfriar para que se gelifique el medio de cultivo. Una vez la mezcla se encuentra en su fase sólida se procede a la inoculación en la superficie, se toman $10 \mu \mathrm{L}$ de cada suspensión 0,5 McFarland, los cuales son depositados en forma de punto sobre el medio; se incuba durante 24 horas a $37^{\circ} \mathrm{C}$. Se realiza un control de crecimiento inoculando $10 \mu \mathrm{L}$ de cada suspendido bacteriano sobre una placa Petri con $10 \mathrm{~mL}$ de agar MuellerHinton (Becton Dickinson, USA); un control de esterilidad dejando en incubación durante 24 horas a $37^{\circ} \mathrm{C}$ una placa Petri con $10 \mathrm{~mL}$ del agar MuellerHinton previamente preparado sin sembrar. Como control del método se utiliza el antibiótico de uso comercial Amoxicilina (SIGMA) partiendo de la concentración $512 \mu \mathrm{g} \mathrm{mL}^{-1}$ hasta la concentración de $2 \mu \mathrm{g} \mathrm{mL}^{-1}$ según la «Preparación de diluciones de agentes antimicrobianos para su uso en pruebas de susceptibilidad a la dilución de agar», disponible en la guía CLSI M100 E28 [31]. Se realiza cada análisis por triplicado.

Se determinó la CMI de dos detergentes desinfectantes de uso comercial producidos por la empresa Spartan Chemical Campany, Inc., Clean By Peroxy a base de peróxido de hidrógeno y Super HDQ Neutral a base de amonios cuaternarios para ser comparada con la CMI de F1. Primero se prepararon los desinfectantes comerciales en 
la dilución recomendada en la etiqueta, 1:32 para Clean By Peroxy y 1:250 para Super HDQ Neutral; se utilizó como agente diluyente agua destilada. Se realizan la dilución en agar para determinar la CMI de cada producto sobre el mismo grupo de bacterias anteriormente probadas. Para evaluar el efecto de la temperatura sobre las formulaciones, teniendo en cuenta que se añaden al agar en fase líquida que se encuentra a $50^{\circ} \mathrm{C}$, se determinó el efecto directo de la nanoemulsión a una temperatura de $30^{\circ} \mathrm{C}$ sobre las bacterias seleccionadas.

\section{Determinación de concentración mínima bactericida (CMB)}

A partir de la placa Petri que contiene el agar con la dilución de formulado que permitió establecer la $\mathrm{MIC}$, se toma una muestra de la superficie de agar con un hisopo estéril y se siembra en un nuevo agar Mueller-Hinton estéril; se incuba durante 48 horas a $37^{\circ} \mathrm{C}$ y se realiza la lectura.

\section{Análisis estadístico}

Para la sistematización de la información y análisis de los resultados se utilizó el software estadístico SPSS versión 24, licenciado por la Universidad de Antioquia. En este se construyeron las tablas descriptivas de la información. Para cada uno de los formulados y desinfectantes comerciales se aplicó la prueba de Chi-cuadrado para observar la relación entre la concentración del producto y el crecimiento de las bacterias, encontrándose en todos los experimentos el valor de la concentración que permitió el no crecimiento de las bacterias en las tres repeticiones realizadas, sin presentar crecimiento en las concentraciones probadas mayores a la CMI, lo que demuestra estabilidad de los formulados. Posteriormente, se aplicó la prueba t de Student, teniendo como referencia la concentración promedio a la que actuó el formulado F1 (con AgNP y d-limoneno) y se estableció si existían diferencias estadísticamente significativas con las medias de los otros productos evaluados. Se tomaron los valores "p" menores o iguales a 0,05 como estadísticamente significativos en ambas pruebas.

\section{Resultados}

\section{Estabilidad de partículas}

En la tabla 1 se presentan los resultados de caracterización coloidal de las formulaciones. F1 (5,6x10-2 \% d-limoneno, 4,7×10-5 \% AgNP) presentó menor tamaño promedio de partícula, menor polidispersidad y mayor estabilidad electrostática.

Tabla 1. Caracterización coloidal de formulaciones evaluadas.

\begin{tabular}{cccc}
\hline & $\mathrm{F} 1$ & $\mathrm{~F} 2$ & $\mathrm{~F} 3$ \\
\cline { 2 - 4 } Diámetro promedio $(\mathbf{n m})$ & $81,5 \pm 0,9$ & $116,4 \pm 9,2$ & $133,7 \pm 1.5$ \\
Moda $(\mathbf{n m})$ & $69,5 \pm 1,7$ & $81,8 \pm 4,8$ & $9,4 \pm 2.7$ \\
D10 (nm) & $60,5 \pm 0,5$ & $72,1 \pm 5,6$ & $86,5 \pm 0.8$ \\
D50 (nm) & $75,1 \pm 0,8$ & $99,7 \pm 8,6$ & $121 \pm 3$ \\
D90 (nm) & $107,8 \pm 2,4$ & $186,3 \pm 18,2$ & $198,4 \pm 4,8$ \\
Polidispersidad & 0,0851 & 0,495 & 0,0928 \\
Potencial Zeta $(\mathbf{m V})$ & $-11,6 \pm 0,3$ & $-8,3 \pm 0,4$ & $-5,1 \pm 0,5$ \\
\hline
\end{tabular}

En las figuras 1, 2y 3 ; se presentan las distribuciones de tamaño de partícula de las formulaciones, y se observa el efecto de la funcionalización del d-limoneno in situ en el proceso de síntesis de las AgNP.

Para la estabilidad coloidal de la formulación 1 , se observa una transmisión que permanece constante a lo largo de la altura del vial (Figura 4a) lo que significa que no se evidencia la migración de partículas (cremación o sedimentación) durante las 24 horas de análisis de la muestra. Por otro lado, la figura $4 \mathrm{~b}$ muestra el perfil de retrodispersión en forma absoluta; aunque no se observa punto isosbéstico, no hay variación del tamaño de la partícula ya que el perfil de retrodispersión está dentro del intervalo de $\pm 2 \%$. 


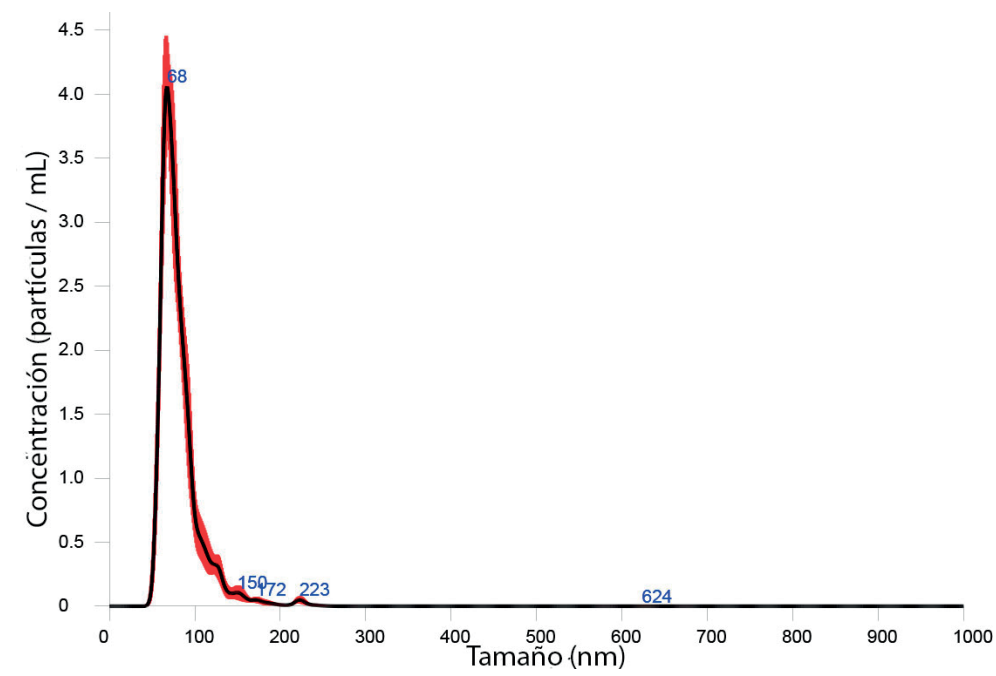

Figura 1. Distribución de tamaño de partícula de la formulación F1.

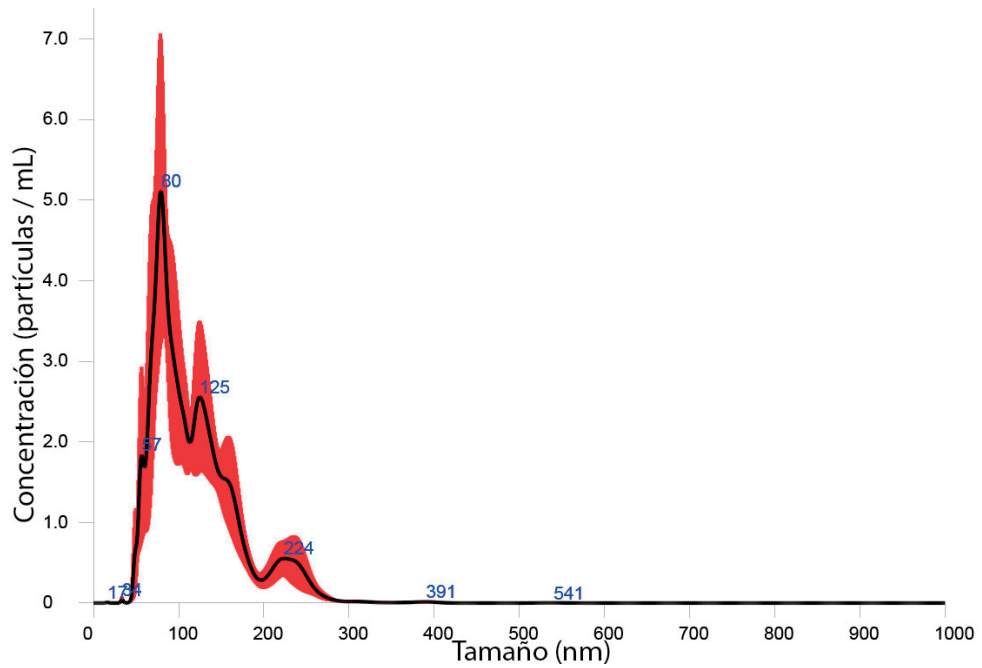

Figura 2. Distribución de tamaño de partícula de la formulación F2.

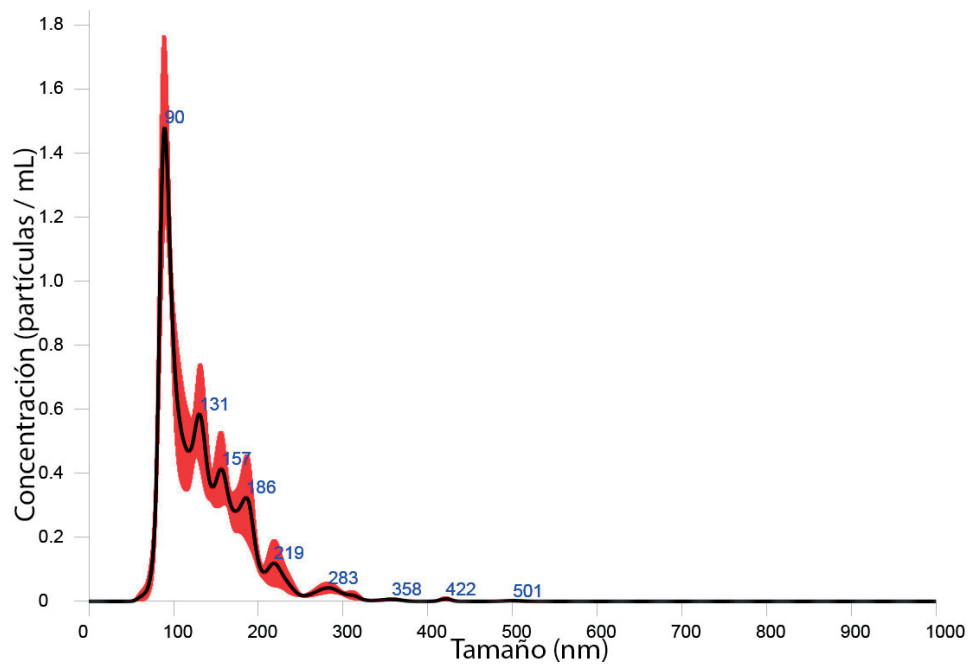

Figura 3. Distribución de tamaño de partícula de la formulación F3. 


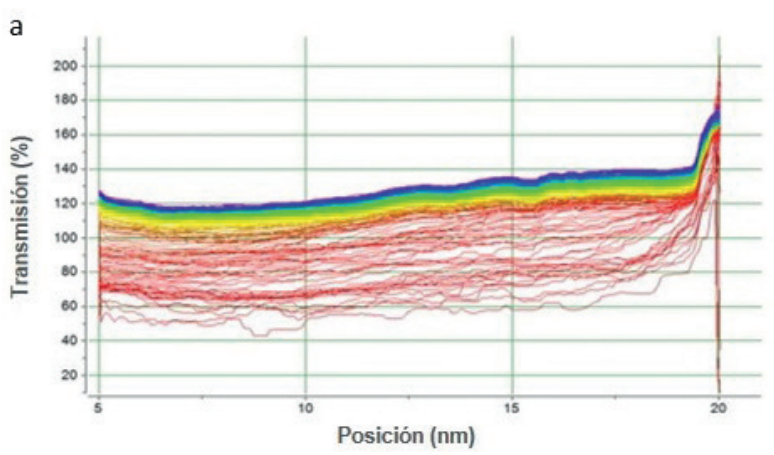

Figura 4. Estabilidad coloidal de la formulación F1: a) Perfil de transmisión; b) Perfil de retrodispersión.

\section{Microbiológicos}

Identificación Bacteriana. Los resultados arrojados por el Vitek 2 Compac indican que todas las identificaciones de las cepas bacterianas alcanzan en promedio un $96 \%$ de probabilidad.

Resultado de la CMI y CMB de los Formulados. La formulación $\mathrm{F} 1$ que contiene AgNP funcionalizadas con d-limoneno presentó una CMI y CMB de $28 \mu \mathrm{g} \mathrm{mL}^{-1}$ con una concentración de d-limoneno y AgNP de $5,6 \times 10^{-2} \%$ y $4,7 \times 10^{-5} \%$, respectivamente, frente a todas las bacterias analizadas; la CMI y CMB para Escherichia coli ATCC 25922, Staphylococcus aureus ATCC 29213, Klebsiella oxytoca ATCC 700324,

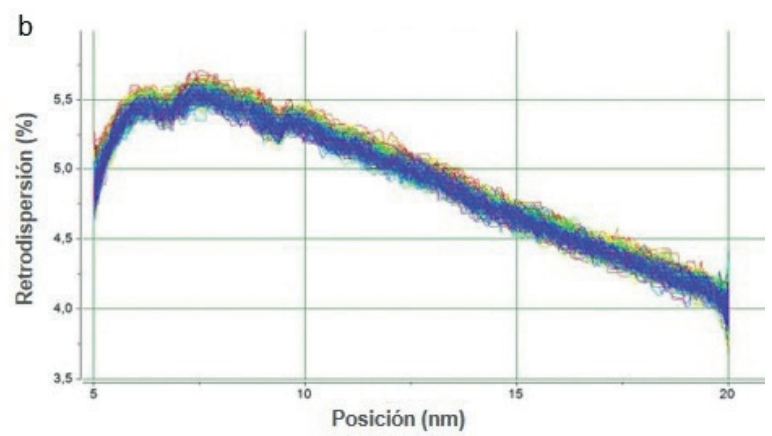

Escherichia coli productora de betalactamasas de espectro extendido BLEE fue de $28 \mu \mathrm{g} \mathrm{mL}^{-1}$, contra Pseudomona aeruginosa fue de $22 \mu \mathrm{g} \mathrm{mL}^{-1}$ y contra Enterococcus casseliflavus fue de $24 \mu \mathrm{g} \mathrm{mL}^{-1}$. En la tabla 2 se encuentran los resultados de cada formulado sobre las bacterias probadas. El control de crecimiento arrojó un resultado positivo en las tres repeticiones y el control del método se reprodujo en el rango esperado de $\mathrm{CMI}$ de la Amoxicilina sobre cada bacteria. Los desinfectantes de uso comercial a base de peróxido de hidrógeno Clean By Peroxy y de amonios cuaternarios Super HDQ Neutral presentaron una CMI de $68 \mu \mathrm{g} \mathrm{mL}^{-1}$ y de $36 \mu \mathrm{g} \mathrm{mL}^{-1}$, respectivamente.

Tabla 2. Concentración mínima inhibitoria/bactericida de los formulados sobre las bacterias probadas.

\begin{tabular}{|c|c|c|c|c|c|c|}
\hline \multirow[b]{2}{*}{ Desinfectante formulado } & \multicolumn{6}{|c|}{ Bacteria - CMI CMB $\mu \mathrm{g} \mathrm{mL^{-1^{* } }}$} \\
\hline & $\begin{array}{l}\text { E.coli } \\
\text { ATCC } \\
25922\end{array}$ & $\begin{array}{l}\text { E.coli } \\
\text { BLEE }\end{array}$ & $\begin{array}{c}P . \text { aeruginosa } \\
R \text { Carb }\end{array}$ & $\begin{array}{l}\text { K. oxytoka } \\
\text { ATCC } \\
700324\end{array}$ & $\begin{array}{c}\text { S. aureus } \\
\text { ATCC } \\
29213\end{array}$ & $\begin{array}{c}\text { E. casseliflavus } \\
\text { ATCC } 700327\end{array}$ \\
\hline F1 AgNP+ d-limoneno & 28 & 28 & 22 & 28 & 28 & 24 \\
\hline F2 Solo AgNP & 30 & 30 & 22 & 34 & 34 & 26 \\
\hline F3 Solo d-limoneno & 40 & 40 & 32 & 46 & 50 & 34 \\
\hline Peróxido de hidrógeno (A) & 68 & 68 & 46 & 46 & 46 & 44 \\
\hline Amonio cuaternario (B) & 20 & 20 & 18 & 36 & 20 & 18 \\
\hline
\end{tabular}

A: Detergente desinfectante a base de peróxido de hidrógeno, Clean by Peroxy, Spartan.

B. Detergente desinfectante a base de amonios cuaternarios para dispositivos médicos, Super HDQ Neutral, Spartan.

* Media de los resultados de tres experimentos diferentes.

El porcentaje de AgNP y d-limoneno en la CMB de los formulados, teniendo en cuenta que la mínima cantidad del producto ensayado para eliminar el total de las bacterias probadas fue de $28 \mu \mathrm{g} \mathrm{mL}-1$ para F1, $34 \mu \mathrm{g} \mathrm{mL}^{-1}$ para F2 y $50 \mu \mathrm{g} \mathrm{mL}^{-1}$ para F3, se reporta en la tabla 3 . 
Tabla 3. Porcentaje de d-limoneno y AgNP en la CMB.

\begin{tabular}{cccc}
\hline & $\mathrm{F} 1$ & $\mathrm{~F} 2$ & $\mathrm{~F} 3$ \\
\cline { 2 - 4 } d-limoneno (\%) & $5,6 \times 10^{-5}$ & $\mathrm{n} / \mathrm{c}^{*}$ & 0,1 \\
AgNP (\%) & $4,7 \times 10^{-5}$ & $5,7 \times 10^{-5}$ & $\mathrm{n} / \mathrm{c}$ \\
\hline
\end{tabular}

En la tabla 4 se puede observar los estadísticos descriptivos para el comportamiento de cada desinfectante. Aquel que tuvo el rango más amplio fue el desinfectante a base de peróxido de hidrógeno, con un valor de $2,4 \mu \mathrm{g} \mathrm{mL} \mathrm{m}^{-1}$. La concentración máxima utilizada en este fue de $68 \mu \mathrm{g} \mathrm{mL}-1$. Aquel que tuvo un rango de acción más pequeño fue la combinación de AgNP y d-limoneno, presentando un valor mínimo de 22 $\mu \mathrm{g} \mathrm{mL}-1$ y máximo de $28 \mu \mathrm{g} \mathrm{mL}^{-1}$, lo cual muestra una mayor estabilidad entre los agentes evaluados. Al observar el comportamiento de la media, se encuentra que los desinfectantes que requirieron en promedio menos volumen para inhibir los agentes evaluados son los desinfectantes a base de amonios cuaternarios, con una media de $22 \mu \mathrm{g} \mathrm{mL}-1$, seguido de F1 (AgNP y d-limoneno) 26,3 $\mathrm{\mu g} \mathrm{mL}^{-1}$. Los dos desinfectantes que tuvieron una CMI mayor fueron F3 (d-limoneno) y el desinfectante a base de peróxido de hidrógeno con medias de $40,3 \mu \mathrm{m} \mathrm{mL}^{-1}$ y $53 \mu \mathrm{g} \mathrm{mL}$ respectivamente.

Tabla 4. Estadísticos descriptivos para el volumen formulado del desinfectante.

\begin{tabular}{ccccccc}
\hline Formulación & $\begin{array}{c}\mathbf{N} \text { (Bacterias } \\
\text { evaluadas })\end{array}$ & $\begin{array}{c}\text { Rango } \\
\left(\mu \mathrm{g} \mathrm{mL}^{-1}\right)\end{array}$ & $\begin{array}{c}\text { Mínimo } \\
\left(\mu \mathrm{g} \mathrm{mL}^{-1}\right)\end{array}$ & $\begin{array}{c}\text { Máximo } \\
\left(\mu \mathrm{g} \mathrm{mL}^{-1}\right)\end{array}$ & $\begin{array}{c}\text { Media } \\
\left(\mu \mathrm{g} \mathrm{mL}^{-1}\right)\end{array}$ & $\begin{array}{c}\text { Desviación } \\
\left(\mu \mathrm{mL}^{-1}\right)\end{array}$ \\
\hline F1 AgNP + d-limoneno & 6 & 0,6 & 22 & 28 & 26,3 & 2,6 \\
$\quad$ F2 Solo AgNP & 6 & 1,2 & 22 & 34 & 29,3 & 4,7 \\
F3 Solo d-limoneno & 6 & 1,8 & 32 & 50 & 40,3 & 6,9 \\
$\begin{array}{c}\text { A. Desinfectante a base de } \\
\text { peróxido de hidrógeno }\end{array}$ & 6 & 2,4 & 44 & 68 & 53 & 12 \\
$\begin{array}{c}\text { B. Desinfectante a base de } \\
\text { amonios cuaternarios }\end{array}$ & 6 & 1,8 & 18 & 36 & 22 & 7 \\
\hline
\end{tabular}

Al tener el comportamiento de las concentraciones promedio necesarias para inhibir el crecimiento de las diferentes bacterias, se realizó la prueba $\mathrm{t}$ de Student teniendo como referencia la media dada para la formulación F1 (AgNP y d-limoneno) $26,3 \mu \mathrm{g} \mathrm{mL}-1$.

Al realizar la prueba $t$ de Student con este parámetro (ver tabla 5), se encuentran diferencias estadísticamente significativas al comparar F1 con F3, la formulación de solo d-limoneno, (valor $p=0,004)$ y el desinfectante a base de peróxido de hidrógeno (valor $p=0,002$ ). Contrariamente, las formulaciones F2 de solo AgNP y los desinfectantes a base de amonio cuaternario tuvieron un comportamiento que no presenta diferencias estadísticamente significativas frente a la formulación de de AgNP+d-limoneno.

Tabla 5. Prueba t de Student de las medias.

\begin{tabular}{ccccc}
\hline \multirow{2}{*}{ Formulación } & \multicolumn{4}{c}{ Valor de prueba = 26,3 } \\
\cline { 2 - 5 } & Prueba $\mathbf{t}$ & gl & Sig. (bilateral) & Diferencia de medias \\
\hline AgNP + limoneno & 0,000 & 5 & 1,000 & 0,0 \\
Solo AgNP & 1,571 & 5 & 0,177 & 3,0 \\
Solo limoneno & 4,999 & 5 & $\mathbf{0 , 0 0 4}$ & 14,0 \\
Desinfectante a base de peróxido de hidrógeno & 5,609 & 5 & $\mathbf{0 , 0 0 2}$ & 26,7 \\
Desinfectante a base de amonios cuaternarios & $-1,532$ & 5 & 0,186 & $-4,3$ \\
\hline
\end{tabular}




\section{Discusión}

Las bacterias analizadas en este estudio son consideradas agentes patógenos para el ser humano; se incluyen cepas de referencia proporcionadas por la empresa American Type Culture Collection ATCC y bacterias que han manifestado in vivo e in vitro mecanismos de resistencia a los antibióticos, como Escherichia coli productora de BLEE, enzimas producidas por las bacterias con la capacidad de inactivar las cefalosporinas de tercera generación (ceftriaxona, cefotaxima, ceftazidima) y el aztreonam [11], Pseudomona aeruginosa con resistencia a los carbapenémicos, grupo de antibióticos que se utilizan para el tratamiento de infecciones causadas por bacterias productoras de BLEE [12]. Las mezclas utilizadas para preparar las nanoemulsiones permitieron lograr una formulación final denominada F1, cuyas propiedades físicas y químicas demuestran que es una mezcla estable, conteniendo partículas con un tamaño promedio de $81,5 \pm 0,9 \mathrm{~nm}$, características que potencian el efecto de la AgNP y el d-limoneno en las pruebas microbiológicas; F1 presentó el mismo efecto sobre $E$. coli silvestre y $E$. coli multirresistente y una $\mathrm{CMI}$ frente a $P$. aeruginosa resistente a los carbapenémicos, menor que la obtenida contra las otras 5 bacterias analizadas; esto sugiere que las AgNP funcionalizadas con d-limoneno se desempeñan con el mismo poder sobre bacterias sin resistencia y bacterias multirresistentes a los antibióticos. La combinación de AgNP con aceites esenciales ya ha demostrado un efecto sinérgico contra bacterias multirresistentes [7].

Se considera la CMI como aquella cantidad de antimicrobiano que, en todos los ensayos, permitió la inhibición completa de crecimiento de todas las bacterias probadas. La formulación denominada F1 demostró una destacada actividad antimicrobiana de amplio espectro ya que actuó de manera similar sobre cocos Gram positivos y bacilos Gram negativos, eliminando el crecimiento in vitro de todas las bacterias probadas con una CMI y CMB de $28 \mu \mathrm{g} \mathrm{mL}^{-1}$ con una media de $26,3 \mu \mathrm{g} \mathrm{mL}^{-1}$; la formulación denominada F2 presento una CMI y una CMB de $34 \mu \mathrm{g} \mathrm{mL}^{-1}$ y la formulación F3 mostro una $\mathrm{CMI}$ y una $\mathrm{CMB}$ de $50 \mu \mathrm{g} \mathrm{mL}^{-1}$; estos datos indican que las AgNP funcionalizadas in situ con d-limoneno en un formulado tipo nanoemulsión presenta un efecto aditivo antimicrobiano, siendo necesario menos cantidad de la formulación F1 para eliminar el 99,9 \% de las bacterias probadas, comparado con F2 y F3. Elementos como Plata $(\mathrm{Ag})$, Oro $(\mathrm{Au})$, Cinc $(\mathrm{Zn})$, Platino $(\mathrm{Pt})$, Hierro $(\mathrm{Fe})$ y Cobre $(\mathrm{Cu})$ se han utilizado en combinación con AE para evaluar su actividad antimicrobiana, evidenciándose un efecto sinérgico [33]. Según el análisis estadístico, F1 presenta valores de CMI significativamente diferentes comparado con F3, formulado que solo contiene d-limoneno, lo que indica que $\mathrm{F} 1$ actúa mejor a menor concentración frente a F3; con F2, que solo contiene AgNP, no se presentan diferencias estadísticamente significativas. A pesar de esto, como se evidencia en la Tabla 4, es necesario menor cantidad del formulado al combinar las AgNP con d-limoneno para inhibir el crecimiento de 5 de las 6 bacterias probadas; el porcentaje de AgNP necesario para inhibir el $100 \%$ de las bacterias probadas en $\mathrm{F} 1$ fue de $4,7 \times 10^{-5} \%$ y el de F2 fue de $5,7 \times 10^{-5} \%$, lográndose una disminución del $1 \times 10^{-5} \%$ al combinar las AgNP con d-limoneno. Esta disminución baja de una unidad puede significar una reducción en la toxicidad del producto para las células eucariotas, lo que refleja que el limoneno actúa como estabilizante de las nanopartículas de plata y, al combinar los dos agentes antibacterianos, no se ve afectado su poder de inhibir crecimiento. El efecto de F1 (AgNP con d-limoneno) comparado con el formulado F2 (solo AgNP) es más notorio sobre $K$. oxytoka y $S$. aureus, bacterias conocidas por su alta patogenicidad y capacidad de generar y transmitir resistencia a los antibacterianos. Este hallazgo es importante pues comprueba el efecto adictivo que genera el limoneno sobre las AgNP.

En las pruebas comparativas del efecto antibacterial de los desinfectantes versus la formulación $F 1$, el desinfectante a base de amonios cuaternarios presentó el mejor efecto inhibitorio frente a las bacterias Escherichia coli ATCC 25922, Staphylococcus aureus ATCC 2921, Enterococcus casseliflavus ATCC 700327, Escherichia coli BLEE+, Pseudomona aeruginosa resistente a carbapenémicos, comparado con la formulación F1 y el desinfectante Clean By Peroxy. Pero la CMI (36 $\left.\mathrm{g} \mathrm{L}^{-1}\right)$ frente a Klebsiella oxytoca ATCC 700324 fue mayor que la CMI $\left(28 \mathrm{~g} \mathrm{~L}^{-1}\right)$ que presentó la formulación $\mathrm{F} 1$ sobre la misma bacteria. Según este dato el formulado F1 se considera con mayor estabilidad en su efecto sobre el total del grupo bacteriano probado, presentando una diferencia significativa con ambos desinfectantes, ya que para eliminar los 6 géneros bacterianos se necesarita mínimo $28 \mu \mathrm{g} \mathrm{mL}^{-1}$ de F1, $36 \mu \mathrm{g} \mathrm{mL}^{-1}$ del amonio cuaternario y $68 \mu \mathrm{g} \mathrm{mL}^{-1}$ de peróxido 
de hidrógeno. El desinfectante a base de peróxido de hidrógeno presentó menor efecto inhibitorio que la formulación $\mathrm{F} 1$ sobre todas las bacterias probadas. Estos hallazgos permiten sugerir que F1 tiene una acción de amplio espectro como agente desinfectante al actuar de modo parejo y en bajas concentraciones sobre bacterias Gram positivas y Gram negativas. Al analizar los valores mínimos y máximos, se nota que el formulado que tuvo un rango de acción más pequeño fue la combinación de AgNP y d-limoneno, presentando un valor mínimo de $22 \mu \mathrm{g} \mathrm{mL}-1$ y máximo de $28 \mu \mathrm{g} \mathrm{mL}^{-1}$, lo cual muestra una mayor estabilidad entre los agentes evaluados. F1 se comportó de manera muy similar al desinfectante comercial a base de amonio cuaternario; este producto es utilizado en lugares como laboratorios, unidades de cuidados intensivos, industrias alimentarias entre otros para controlar patógenos peligrosos, por lo cual, se concluye que F1 presenta un potencial como agente desinfectante que está a la altura de los desinfectantes de última generación con la ventaja que su efecto es más parejo sobre un grupo heterogéneo de bacterias comparado con el amonio cuaternario.

La combinación de AgNP con AE para alcanzar mayor efecto antimicrobiano contra bacterias ha sido probada en varios estudios. Se ha reportado el efecto aditivo entre AgNP y el terpeno timol para desinfectar el tejido vegetativo de planta Bermudagrass [34]. Las AgNP combinadas con el aceite esencial de Oreganum spp presentaron un efecto adictivo contra bacterias multirresistentes [32]. La mezcla de AgNP con el $\mathrm{AE}$ de Oreganum spp. presentó estabilidad antimicrobiana contra bacterias Gram positivas [35]; AgNP funcionalizadas con aceites esenciales de las plantas Cymbopogon citratus, C. martini, Eucalyptus globules, Azadirachta indica, Ocimum sanctum presentaron efecto contra la bacteria $S$. aureus [36]. Este es el primer reporte del efecto antibacteriano in vitro de una mezcla de AgNP y d-limoneno; los resultados sugieren que el efecto aditivo de las AgNP funcionalizadas con d-limoneno puede ser usado para evitar el crecimiento de bacterias incluyendo multirresistentes que son consideradas patógenas para el ser humano y otras especies; se hace necesario llevar a cabo investigaciones adicionales sobre combinaciones de AgNP con productos de origen natural para brindar más alternativas contra el fenómeno de multirresistencia bacteriana.

Los mecanismos de acción de los AE sobre las células bacterianas varían dependiendo de su composición y de la cepa bacteriana expuesta; los AE tienen como características su hidrofobicidad y la naturaleza lipofílica, que les permite interactuar fácilmente con los ácidos grasos de la membrana celular microbiana; actúan sobre la integridad de la membrana celular al cambiar la permeabilidad, lo que conduce a fugas de electrolitos y pérdida de contenido intracelular vital como proteínas, reduciendo azúcares, mientras que inhibe la generación de energía, conduciendo a la destrucción de las células [37-40]. El d-limoneno derivado del aceite esencial de los cítricos actúa sobre las membranas citoplasmáticas de los microorganismos, causando una pérdida de integridad de la membrana, la inhibición de las enzimas respiratorias y disipación de la fuerza motriz de protones [41].

El mecanismo de acción de las AgNP sobre las bacterias inicia con la unión a la membrana celular, aumentando la permeabilidad, produciendo la liberación de lipopolisacáridos, proteínas de membrana y la unión posterior a los ácidos nucleicos, bloquean las cadenas respiratorias, generando especies reactivas de oxígeno, que conducen a cambios funcionales en la célula llevándola a la muerte [3,5,21-23,25,33,42]. El efecto depende del área de superficie que se encuentra aumentada por la presentación en tamaño nanométrico, pudiendo interactuar en mayor proporción con moléculas como enzimas y ácidos nucleicos, causando mayores cambios estructurales y deformación en las paredes y membranas bacterianas $[2,21,43]$. Por otro lado, algunos autores reportan cepas bacterianas que presentan mecanismos de adaptación y o resistencia a AgNP [21, 23], por lo que es importante continuar evaluando si la mezcla de AgNP con aceites esenciales con poder antibacteriano con el d-limoneno contrarresta el efecto de resistencia que se ha presentado sobre las AgNP. Sin embargo, los resultados evidencian que, la adición de d-limoneno a la formulación requiere menor cantidad de AgNP lo que disminuye la toxicidad por presencia de plata en células eucariotas.

Según los mecanismos de acción de los AE y las AgNP, se puede sugerir que la nanoemulsión F1 presenta un mecanismo de acción combinado y de sinergia; el d-limoneno por su naturaleza lipofílica interactúa fácilmente con los ácidos grasos de la membrana celular microbiana, dañando la integridad de la membrana, las AgNP también afectan la membrana, permitiendo el fácil ingreso de las AgNP 
y del limoneno al citoplasma celular, allí interrumpen el proceso de transporte de electrones inhibiendo la secreción de toxinas al medio ambiente, causando disfunción de los ribosomas, interactúan con el material genético hasta degradarlo y lograr, finalmente, la lisis celular [33].

\section{Conclusiones}

El problema de la resistencia bacteriana crece de manera alarmante, aumentando considerablemente las tasas de morbilidad y mortalidad a nivel mundial. El nivel de evolución de las bacterias para sobrevivir y multiplicarse en ambientes con altas concentraciones de antibióticos y desinfectantes disponibles comercialmente, en la última década, sucede a una tasa mucho mayor que la evolución en el desarrollo de antimicrobianos por parte de los científicos. Es necesario el desarrollo de nuevos productos que demuestren un efecto microbicida sobre todo tipo de bacterias patógenas, especialmente las que presentan mayores mecanismos de resistencia. Las AgNP son consideradas como una real alternativa para el desarrollo de antimicrobianos contra bacterias multirresistentes por su alta toxicidad para las células bacterianas. Sin embargo, se han reportado bacterias que presentan mecanismos de resistencia a las AgNP y se hace importante, entonces, probar mezclas de las AgNP con otros agentes que presentan potencial microbicida en búsqueda de sinergia, para alcanza un mejor efecto antibacteriano y contrarrestar la resistencia. Algunos derivados de AE han demostrado efecto antibacteriano de amplio espectro; el d-limoneno como componente principal del $\mathrm{AE}$ de los cítricos presentó un buen efecto bactericida a bajas concentraciones, convirtiéndose en una alternativa para combatir gérmenes de importancia clínica.

La formulación que contiene AgNP funcionalizadas con d-limoneno produce un efecto aditivo para eliminar el crecimiento de bacterias patógenas, comparada con la nanoemulsión que contenía solo AgNP o la nanoemulsión que solo contenía limoneno. F1 presentó un efecto similar al obtenido con desinfectantes de uso comercial; lo anterior sugiere que las AgNP se pueden potenciar al mezclarse con aceites esenciales y así ofrecer mejores efectos contra la integridad de la célula bacteriana.

\section{Agradecimientos}

Los autores expresan su agradecimiento a la Universidad EIA, a la Fundación de Apoyo a la Investigación en el Grupo Interdisciplinario de Estudios Moleculares-FUNDAGIEM de la Universidad de Antioquia y al Laboratorio Clínico Hematológico.

\section{Referencias bibliográficas}

[1] World Health Organization. Prioritization of pathogens to guide discovery, research and development of new antibiotics for drugresistant bacterial infections, including tuberculosis. Switzerland: World Health Organization; 2017.

[2] Ansari M, Khan H, Khan A, Cameotra S, Saquib $Q$, Musarrat J. Gum arabic cappedsilver nanoparticles inhibit biofilm formation by multi-drug resistant strains of Pseudomonas aeruginosa. J. Basic Microbiol. 2014;54(7):688-99. https://doi.org/10.1002/ jobm.201300748

[3] Rai M, Deshmukh S, Ingle A, Gade A. Silver nanoparticles: the powerful nanoweapon against multidrug-resistant bacteria. J. Appl. Microbiol. 2012;112(5):841-52. https://doi. org/10.1111/j.1365-2672.2012.05253.x

[4] Simões D, Miguel S, Ribeiro M, Coutinho P, Mendonça A, Correia I. Recent advances on antimicrobial wound dressing: A review. Eur. J. Pharm. Biopharm. 2018;127:130-41. https:// doi.org/10.1016/j.ejpb.2018.02.022

[5] Pérez ZC, Torres C, Nuñez M. Antimicrobial Activity and Chemical Composition of Essential Oils from Verbenaceae Species Growing in South America. Molecules. 2018;23(3):544. https://doi.org/10.3390/molecules23030544

[6] World Health Organization. Antibacterial agents in clinical development: an analysis of the antibacterial clinical development pipeline, including tuberculosis. Switzerland: World Health Organization; 2017.

[7] Sheikholeslami S, Mousavi S, Ahmadi AH, Hosseini DS, Mahdi RS. Antibacterial Activity of Silver Nanoparticles and Their Combination with Zataria multiflora Essential Oil and Methanol Extract. Jundishapur J. Microbiol. 2016;9(10):e36070. https://doi.org/10.5812/ jjm.36070 
[8] Kaviya S, Santhanalakshmi J, Viswanathan B, Muthumary J, Srinivasan K. Biosynthesis of silver nanoparticles using citrus sinensis peel extract and its antibacterial activity. Spectrochim. Acta, Part A. 2011;79(3):594-98. https://doi.org/10.1016/j.saa.2011.03.040

[9] Ministerio de Salud. Plan Nacional de Respuesta a la Resistencia a los Antimicrobianos. Plan Estratégico Dirección de Medicamentos y Tecnologías en Salud. Ministerio de Salud. Colombia: Ministerio de Salud; 2018.

[10] Organización Mundial de la Salud. Sistema mundial de vigilancia de la resistencia a los antimicrobianos. Manual para la primera fase de implementación. Suiza: Organización Mundial de la Salud; 2017.

[11] Morejón García M. Betalactamasas de espectro extendido. Rev. Cubana Med. 2013;52(4):272-80.

[12] Suarez C, Kattan J, Guzmán A, Villegas M. Mecanismos de resistencia a carbapenems en $P$. aeruginosa, Acinetobacter y Enterobacteriaceae y estrategias para su prevención y control. Infectio. 2006;10(3):8593.

[13] Torrenegra M, Pájaro N, Méndez L. Actividad antibacteriana in vitro de aceites esenciales de diferentes especies del género Citrus. Rev. Colomb. Cienc. Quim.-Farm. 2017;46(2):16075.https://doi.org/10.15446/rcciquifa. v46n2.67934

[14] Shao P, Zhang H, Niu B, Jiang L. Antibacterial activities of $\mathrm{R}$-(+)-Limonene emulsion stabilized by Ulva fasciata polysaccharide for fruit preservation. Int. J. Biol. Macromol. 2018;111:1273-80. https://doi.org/10.1016/j. ijbiomac.2018.01.126

[15]Mitropoulou G, Fitsiou E, Spyridopoulou K, Tiptiri-Kourpeti A, Bardouki H, Vamvakias $M$, et al. Citrus medica essential oil exhibits significant antimicrobial and antiproliferative activity. LWT-Food Sci. Technol. 2017;84:34452. https://doi.org/10.1016/j.Iwt.2017.05.036

[16]Pekmezovic M, Aleksic I, Barac A, ArsicArsenijevic V, Vasiljevic B, Nikodinovic-Runic $\mathrm{J}$, et al. Prevention of polymicrobial biofilms composed of Pseudomonas aeruginosa and pathogenic fungi by essential oils from selected Citrus species. Pathog. Dis. 2016;74(8):ftw102. https://doi.org/10.1093/femspd/ftw102

[17] Montironi I, Cariddi L, Reinoso E. Evaluation of the antimicrobial efficacy of Minthostachys verticillata essential oil and limonene against Streptococcus uberis strains isolated from bovine mastitis. Rev. Argent. Microbiol. 2016;48(3):210-16. https://doi.org/10.1016/j. ram.2016.04.005

[18]Chen G, Lin Y, Lin C, Jen H. Antibacterial Activity of Emulsified Pomelo (Citrus grandis Osbeck) Peel Oil and Water-Soluble Chitosan on Staphylococcus aureus and Escherichia coli. Molecules. 2018;23(4):840. https://doi. org/10.3390/molecules23040840

[19]Lou Z, Chen J, Yu F, Wang H, Kou X, Ma C, et al. The antioxidant, antibacterial, antibiofilm activity of essential oil from Citrus medica L. var. sarcodactylis and its nanoemulsion. LWT. 2017;80:371-7. https://doi.org/10.1016/j. Iwt.2017.02.037

[20]Al-Aamri M, Al-Abousi N, Al-Jabri S, Alam T, Khan S. Chemical composition and in-vitro antioxidant and antimicrobial activity of the essential oil of Citrus aurantifolia $L$. leaves grown in Eastern Oman. J Taibah Univ Med Sci. 2018;13(2):108-12. https://doi.org/10.1016/j. jtumed.2017.12.002

[21] Rudakiya D, Pawar K. Bactericidal potential of silver nanoparticles synthesized using cell-free extract of Comamonas acidovorans: in vitro and in silico approaches. 3 Biotech. 2017;7:92. https://doi.org/10.1007/s13205-017-0728-3

[22]Abdel-Aziz M, Shaheen M, El-Nekeety $A$, Abdel-Wahhab M. Antioxidant and antibacterial activity of silver nanoparticles biosynthesized using Chenopodium murale leaf extract. J. Saudi Chem. Soc. 2014;18(4):356-63. https:// doi.org/10.1016/j.jscs.2013.09.011

[23] McQuillan J, Groenaga IH, Stokes E, Shaw A. Silver nanoparticle enhanced silver ion stress response in Escherichia coli K12. Nanotoxicology. 2011;6(8):857-866. https:// doi.org/10.3109/17435390.2011.626532

[24] Guzman M, Dille J, Godet S. Synthesis and antibacterial activity of silver nanoparticles against gram-positive and gram-negative bacteria. Nanomedicine. 2012;8(1):37-45. https://doi.org/10.1016/j.nano.2011.05.007

[25] Vilas V, Philip D, Mathew J. Essential oil mediated synthesis of silver nanocrystals for environmental, anti-microbial and antioxidant applications. Mater. Sci. Eng. 2016;61:429-36. https://doi.org/10.1016/j.msec.2015.12.083

[26] Ramírez LS, Marin Castaño D. Metodologías para evaluar in vitro la actividad antibacteriana de compuestos de origen vegetal. Scientia et 
Technica. 2009;2(42):263-8.

[27] Herrera ML. Pruebas de sensibilidad antimicrobiana Metodología de laboratorio. Rev. méd. Hosp. Nac. Niños. 1999;34:33-41.

[28] Jiménez N, Cienfuegos A, González G, Higuita L. Medios de cultivo, pruebas de identificación y pruebas de susceptibilidad. Medellín, Colombia: Universidad de Antioquia; 2015.

[29] Picazo JJ. Procedimientos en Microbiología Clínica. Recomendaciones de la Sociedad Española de Enfermedades Infecciosas y Microbiología Clínica. Métodos básicos para el estudio de sensibilidad a los antimicrobianos. España: Sociedad Española de Enfermedades Infecciosas y Microbiología Clínica; 2000.

[30] CLSI M07 - Methods for Dilution Antimicrobial Susceptibility Tests for Bacteria That Grow Aerobically. 11 Edition. Clinical and Laboratory Standards Institute. 2018.

[31]CLSI. M100 Performance standards for antimicrobial susceptibility testing. 28 Edition. United States: Clinical and Laboratory Standards Institute; 2018.

[32] Scandorieiro S, Camargo L, Lancheros C, Yamada-Ogatta, S, Nakamura, C, Oliveira, A, et al. Synergistic and additive effect of oregano essential oil and biological silver nanoparticles against multidrug-resistant bacterial strains. Front. Microbiol. 2016;7:760. https://doi. org/10.3389/fmicb.2016.00760

[33] Raia M, Paralikara P, Jogeea P, Agarkara $\mathrm{G}$, Inglea $\mathrm{A}$, Deritab $\mathrm{M}$, et al. Synergistic antimicrobial potential of essential oils in combination with nanoparticles: Emerging trends and future perspectives. Int $\mathrm{J}$ Pharm. 2017;519(1-2):67-78. https://doi.org/10.1016/j. ijpharm.2017.01.013

[34] Taghizadeh M, Solgi M. The application of essential oils and silver nanoparticles for sterilization of Bermuda grass explants in in vitro culture. Int. J. Hortic. Sci. Technol. 2014;1(2):131-40. https://doi.org/10.22059/ IJHST.2014.52784

[35] Khalaf $H$, Sharoba A, El-Tanahi H, Morsy M. Stability of antimicrobial activity of pullulan edible films incorporated with nanoparticles and essential oils and their impact on turkey deli meat quality. J. Food Dairy Sci. Mansoura Univ. 2013;4(11):557-73. https://doi.org/10.21608/ jfds.2013.72104
[36]Bansod S, Bawaskar M, Gade A, Rai M. Development of shampoo, soap and ointment formulated by green synthesized silver nanoparticles functionalized with antimicrobial plants oils in veterinary dermatology: treatment and prevention strategies. IET Nanobiotechnol. 2015;9:165-71. https://doi.org/10.1049/ietnbt.2014.0042

[37] Cui H, Zhang X, Zhou H, Zhao C, Lin L. Antimicrobial activity and mechanisms of Salvia sclarea essential oil. Bot. Stud. 2015;56(16):28. https://doi.org/10.1186/s40529-015-0096-4

[38] Huang D, Xu J, Liu J, Zhang H, Hu Q. Chemical constituents, antibacterial activity and mechanism of action of the essential oil from Cinnamomum cassia bark against four food related bacteria. Microbiology 2014;83:357-65. https://doi.org/10.1134/S0026261714040067

[39] Li C, Yu J. Chemical composition: antimicrobial activity and mechanism of action of essential oil from the leaves of Macleaya cordata (Willd). R. Br. J. Food Saf. 2015;35(2):227-36. https:// doi.org/10.1111/jfs. 12175

[40]Lakehal S, Meliani A, Benmimoune S, Bensouna S, Benrebiha F, Chaouia, C. Essential oil composition and antimicrobial activity of Artemisia herba- alba Asso grown in Algeria. Med. Chem. (Los Angeles). 2016;6(6):435-9. https://doi.org/10.4172/21610444.1000382

[41]Zhang Z, Vriesekoop F, Yuan Q, Liang $H$, Effects of nisin on the antimicrobial activity of D-limonene and its nanoemulsión. Food Chem. 2014;150:307-12. https://doi.org/10.1016/j. foodchem.2013.10.160

[42] Mohamed A, Mohamed S, Aziza E, Mosaad A. Antioxidant and antibacterial activity of silver nanoparticles biosynthesized using Chenopodium murale leaf extract. J. Saudi Chem. Soc. 2014;18:356-63. https://doi. org/10.1016/j.jscs.2013.09.011

[43]Rhim J, Wang L, Hong S. Preparation and characterization of agar/silver nanoparticles composite films with antimicrobial activity. Food Hydrocolloids. 2013;33(2):327-35. https://doi.org/10.1016/j.foodhyd.2013.04.002

[44] Katz L, Baltz R. Natural product discovery: past, present, and future. J Ind Microbiol Biotechnol. 2016;43(2-3):155-76. https://doi. org/10.1007/s10295-015-1723-5 